\title{
La notoriedad de la marca como factor explicativo de la eficacia de las campañas publicitarias en televisión
}

The Brand Awareness as a Factor Explaining the Effectiveness of Television Advertising

Josefa D. Martín-Santana

Universidad de Las Palmas de Gran Canaria josefa.martin@ulpgc.es

Pedro Reinares-Lara

Universidad Rey Juan Carlos

pedro.reinares@urjc.es

Eva Reinares-Lara

Universidad Rey Juan Carlos

peva.reinares@urjc.es
Martín-Santana, J. D. , Reinares-Lara, P., Reinares-Lara, E. (2015), La notoriedad de la marca como factor explicativo de la eficacia de las campañas publicitarias en televisión.

Revista Internacional de Investigación en Comunicación aDResearch ESIC. No 11 Vol 11.

Primer semestre, Enero-junio 2015 · Págs. 8 a 25

DOI: 11.7263/ADRESIC.011.001 
RESUMEN

\section{Clasificación JEL: \\ M1, M3}

Palabras clave:

Recuerdo publicitario, eficacia publicitaria, valor de marca, notoriedad de marca, publicidad en televisión.
El propósito de esta investigación es analizar el poder explicativo de la notoriedad de marca en la eficacia de las campañas publicitarias en el medio televisión medida en términos de recuerdo espontáneo de marca. Para ello se ha utilizado un análisis de regresión jerárquica en el que (1) la variable dependiente ha sido el recuerdo publicitario medido en un entorno real y con una muestra representativa de la audiencia de televisión en España y (2) una de las variables independientes eran los resultados de la notoriedad previa de las marcas anunciadas. Las variables de control incluidas han sido la duración del anuncio, la posición del mismo y el número de inserciones. De esta manera se han proporcionado las evidencias empíricas para justificar el potencial de la notoriedad de marca en la práctica publicitaria. Estas evidencias demuestran, por un lado, que el grado de notoriedad previa de la marca comunicada afecta positivamente al recuerdo de campañas posteriores, y su vez, la presión publicitaria del sector en el que opera la marca en el medio televisión ejerce también una influencia positiva en los porcentajes de recuerdo de la misma. La importancia de este estudio es clave en la actual situación del medio televisión que está provocando una pérdida de eficacia general de la publicidad, que ha propiciado la necesidad de plantear el papel que tienen actualmente estas variables sobre el recuerdo, el cual, a su vez, se puede ver afectado por la existencia de otros factores considerados de forma parcelada en la literatura, y que en nuestro estudio se han considerado conjuntamente como variables de control.

The purpose of this investigation is to analyze the explanatory power of brand awareness in effectiveness of television advertising measured in terms of unaided recall. This study uses a hierarchical regression analysis in which (1) the dependent variable was advertising recall, measured in a real environment with a representative sample of television audience in Spain and (2) one of the independent variables was the results of the previous awareness of the advertised brands. The variables of control included: duration of the advertisement, position and number of insertions. Thus we have provided empirical evidence to justify the potential of brand awareness in advertising practice. These evidences show that the degree of brand awareness affects the recall of advertising, and in turn, the advertising sector on television also has a positive influence on the percentages of memory of it. 


\section{Introducción}

La importancia del medio televisión en las estrategias de comunicación de los anunciantes tiene su explicación en la elevada tasa de penetración de este medio en la sociedad actual, lo que permite obtener tasas elevadas de cobertura en los diferentes targets (Zenith, 2013). Se trata de un medio estable que ha resistido al avance de nuevos medios (principalmente Internet) y a la creación de nuevos soportes y formas de visionado. Sus cualidades como medio publicitario están bien documentadas (Lowrey, Shrum y Mccarty, 2005; Jamhouri y Winiarz, 2009; Rubinson, 2009). Sin embargo, su actual entorno competitivo le asocia unas limitaciones que hacen que reiteradamente se cuestione su eficacia desde cada uno de los agentes implicados en la comunicación comercial (Davies y Rojas-Mendez, 2005; Mediaedge, 2008), debido a: (1) la fragmentación de las audiencias
(Sharp, Beal y Collins, 2009), lo que genera aumento de costes, dificultad para conseguir coberturas netas altas, complejidad para elegir las distintas opciones o incertidumbre en los resultados del plan de medios; (2) el zapping, que ha provocado un cambio importante en el diseño y colocación de anuncios televisivos (Gázquez, Jiménez, Sáez y Sánchez, 2010), además de reducir su eficacia (Tse y Lee, 2001; Woltman Elpers, Wedel y Pieters, 2003); (3) las nuevas formas de consumo televisivo a través de medios como el ordenador o el móvil (Reinares, 2010), y (4) la elevada saturación del medio, a pesar de la caída de la inversión publicitaria en televisión, como lo demuestra el hecho de que el número de spots emitidos apenas presente variaciones en la actualidad (Zenith, 2013).

A pesar de estas limitaciones, que ponen en evidencia su eficacia, los datos de la Figura 1 indican que proporciona elevados niveles de notorie-

\section{Figura 1 · Cuotas de inversión-notoriedad por medios. Año 2012}

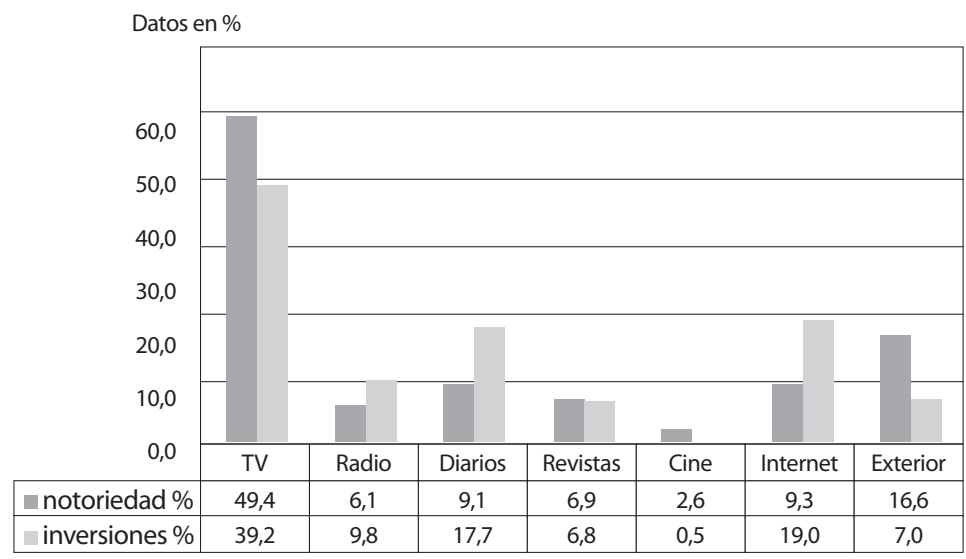


dad de marca, que es una de las dimensiones del valor de la marca.

Keller y Aaker (1997) y Del Río Lanza, Iglesias Argüelles y Vázquez Casielles (2000) señalan que la marca es un recurso intangible susceptible de generar ventajas competitivas y surge de los componentes que aportan valor al consumidor (Feldwick, 1996; Villarejo Ramos, 2002; Ruževičiūtè y Ruževičius, 2010), de ahí que su medición sea considerada desde una óptica multidimensional (Villarejo Ramos, 2002; Del Río Lanza, Iglesias Argüelles y Vázquez Casielles, 2002).

La importancia de la notoriedad en la conformación de la marca se pone de manifiesto en el modelo de cinco dimensiones de Aaker (1991), la cual ha sido reconocida por Keller (1993) y determinado su peso en el modelo por Ruževičiūtè y Ruževičius (2010).

De acuerdo con Lambin (1995, p. 141), la notoriedad es un atributo de la marca de gran importancia para cualquier empresa, ya que un grado elevado de notoriedad constituye un activo importante que se logra a lo largo de años y que exige una inversión publicitaria importante y regular. Se trata de un valor en sí misma, ya que una marca será más importante cuanto más notoria sea (Carrero y González, 2008). De ahí que en la literatura académica se observe la preocupación por contrastar las funciones que cumple la notoriedad. Entre ellas cabe señalar su influencia sobre la mejora de la calidad percibida (Hoyer y Brown, 1990) y su correlación con el liderazgo de la marca bajo ciertas circunstancias (Keller, 1993). En este mismo sentido, Kotler y Keller (2007) manifiestan la importancia del efecto diferencial de las marcas conocidas, notorias, sobre la respuesta del consumidor a los esfuerzos de marketing de la marca. En este contexto, la notoriedad puede jugar un papel relevante en la eficacia de las campañas publicitarias, siendo, por tanto, necesario analizar en qué medida la notoriedad puede considerarse como un factor explicativo de las respuestas de los consumidores a los estímulos publicitarios. Entre estas respuestas, el recuerdo publicitario que genera la publicidad es una de las medidas de eficacia de mayor relevancia, fundamentándose en la creencia de que la publicidad es más eficaz en la medida en que genera un mayor recuerdo, ya que en estas circunstancias es mayor la probabilidad de que se produzca un cambio de actitud y se consiga la conducta deseada (Dubow, 1994). Cómo aumentar la eficacia de las campañas ha sido y seguirá siendo uno de los temas cruciales y más frecuentemente estudiados en la investigación publicitaria (Lamas, 2004).

En este contexto, se refuerza la necesidad de realizar trabajos que desvelen cómo aumentar la eficacia publicitaria y la función que puede ejercer la notoriedad de la marca en el recuerdo de las campañas, como una extensión más a las funciones de la notoriedad de marca proporcionadas en la literatura. Sin embargo, a pesar de que la teoría del marketing propone que la notoriedad de marca puede ser un activo muy valioso para una empresa, la investigación desarrollada por Macdonald y Sharp (2003) concluye que la praxis empresarial concede poca relevancia al mantenimiento y supervisión de la notoriedad de la marca.

Sobre la base de lo anteriormente expuesto, el objetivo de este trabajo es analizar el poder explicativo de una de las dimensiones del valor de la marca (la notoriedad «previa» de marca) en la eficacia de las campañas publicitarias en televisión medida en términos de recuerdo espontáneo de marca. 


\section{El recuerdo publicitario}

\subsection{El recuerdo como medida de eficacia publicitaria}

Las técnicas cognoscitivas de medición de la eficacia publicitaria, en las que se encuadra el recuerdo, son de gran interés y deben ser utilizadas para cualquier campaña, independientemente de su objetivo, pues como afirma Lambin (1995, p. 542) «Una información no existe para el comprador mientras no haya sido percibida, comprendida y memorizada por él». Esta misma idea ha sido compartida por autores como Aaker, Batra y Myers (1992), Rossiter y Percy (1997) o Wells, Burnett y Moriarty (2005).

Entre las técnicas cognoscitivas destacan las medidas de memoria, con las que se evalúa la intensidad del impacto de un mensaje a través de la capacidad del público para recordarlo y reconocerlo, ya que una vez asegurada la exposición del individuo al anuncio, la percepción del mismo se considera como un requisito primordial para que puedan tener lugar los restantes efectos, tales como despertar interés, transmitir lo que se desea, y formar en el individuo una actitud favorable (Higie y Sewall, 1991; Wells et al., 2005).

Para medir el nivel de aprendizaje que generan los mensajes se utiliza tanto el recuerdo como el reconocimiento (Bendixen, 1993; Perreault y Pettigrew, 1998; Beerli y Martín, 1999). A pesar de que ambas técnicas permiten medir la memoria explícita (Shapiro y Krishnan, 2001), el reconocimiento se ha utilizado más en el medio prensa y el recuerdo en televisión (Zhielske, 1982). No obstante, el reconocimiento se percibe como una medida poco fiable, cuyas puntuaciones pueden llegar a ser indiscriminadamente altas, perdiéndose su capacidad para diferenciar entre los distintos niveles de aprendizaje genera- dos por las campañas. Por ello y dado que este trabajo se ha centrado en campañas televisivas, se ha optado por el recuerdo como medida de eficacia cognoscitiva y, más concretamente, el recuerdo espontáneo, ya que, como señala Aaker et al. (1992), una marca es recordada cuando es citada correctamente sin la necesidad de mencionar ni la categoría de producto ni un conjunto de marcas para que el consumidor elija la recordada.

\subsection{Factores condicionantes del recuerdo}

Beerli y Martín (1999) han agrupado los factores que influyen en los procesos memorísticos en tres grandes categorías relacionados con la planificación publicitaria, el producto anunciado y las características del individuo, incluyéndose la familiaridad de la marca (notoriedad previa) en la segunda de ellas. En esta línea, Keller (1993), Kent y Allen (1994) y Pieters, Warlop y Wedel (2002) señalan que la familiaridad de la marca para las audiencias (notoriedad previa) es una variable que influye en el recuerdo publicitario. La notoriedad de marca, que se mide genéricamente o en investigaciones puntuales a lo largo del transcurso de la campaña, desarrolla un efecto acumulativo que se mantiene incluso al finalizar la misma, generando un efecto residual importante (Mela, Gupta y Lehmann, 1997). En línea con este discurso, es coherente plantear que la presión publicitaria anterior aplicada sobre la marca también puede ejercer una influencia directa sobre el recuerdo.

Sin embargo, a pesar del consenso existente sobre la influencia de estas variables en el recuerdo, algunas fuentes afirman que estos efectos positivos sobre el recuerdo se pueden ver mermados por la existencia de otros factores o estímulos que capten una mayor atención en las 
audiencias y, por tanto, un mayor recuerdo (Balasubramanian, Karrh y Patwardhan, 2006). Así, por ejemplo, una marca o una creatividad nueva puede llegar a recibir más atención por su carácter novedoso. Si esto es así, las marcas generarían un rendimiento decreciente en su notoriedad a partir de un cierto nivel de presión publicitaria debido al efecto reactancia, que se produce porque a altos niveles de exposición las audiencias se aburren e irritan, lo que genera niveles de recuerdo decrecientes. Si bien esta teoría tuvo mucha aceptación en los años 80 , los objetivos de comunicación de los anunciantes que más invierten en televisión evidencian su desacuerdo con la misma, ya que los anunciantes líderes (con elevados presupuestos) promueven, como uno de sus objetivos, mantener elevados niveles de presión publicitaria. Estas prácticas no parecen injustificadas si se tiene en cuenta que a pesar de las numerosas evidencias empíricas sobre el efecto repetición (rendimiento decreciente de los estímulos publicitarios), otros estudios no lo confirman. Campbell y Keller (2003) recapitulan que las inconsistencias entre eficacia y repetición de los mensajes se deben a que depende de muchos factores (complejidad del mensaje, implicación con la marca, etc.), y entre ellos está la familiaridad con la marca (notoriedad previa). Por ello parece razonable la formulación de las siguientes hipótesis, asumiendo que las prácticas de los anunciantes reconocen el valor de una notoriedad elevada.

H1. El grado de notoriedad previa de la marca afecta positivamente al recuerdo de una campaña.

H2. La presión publicitaria de una marca afecta positivamente al recuerdo de una campaña.

Por otra parte, y siguiendo la clasificación propuesta por Beerli y Martín (1992), el re- cuerdo es una medida de eficacia publicitaria que también es sensible a factores relacionados con la planificación publicitaria tales como la duración del anuncio, la posición del anuncio o el número de inserciones, todos ellos factores que serán considerados en este trabajo como variables de control y que pasamos a explicar brevemente.

Por las implicaciones económicas de la duración del anuncio, el análisis de su relación con los efectos en la audiencia ha sido objeto de varios trabajos, que se han centrado especialmente en los efectos cognitivos medidos en términos de recuerdo (Fabian, 1986; Singh y Cole, 1993; Bigné y Miquel, 1996). En general, se demuestra que los spots de mayor duración modifican mejor la capacidad de aprendizaje que los de menor duración y, por lo tanto, son capaces de generar mayor recuerdo, ya que, de acuerdo con Batra y Ray (1986), la audiencia tiene más tiempo para procesar el contenido de los mensajes publicitarios.

El estudio de la influencia de la primera y última posición en el recuerdo publicitario ha sido objeto de varios trabajos en publicidad convencional (Pieters y Bijmolt, 1997; Zhao, 1997; Moorman, Neijens y Smit, 2005), dando lugar a los denominados efectos primacía y recencia. De ahí que en la práctica se asuma que los mensajes comerciales colocados en primera y última posición dentro de un bloque publicitario son mejor recordados que los colocados en posiciones intermedias, lo cual ha sido contrastado en los trabajos de Newell, Henderson y Wu (2001) y Li (2010). Esta práctica puede sustentarse sobre la base de dos antecedentes teóricos. Por una parte, el proceso de interferencia entre los diferentes mensajes contribuye a explicar la primacía y la recencia en el recuerdo de los anuncios en tele- 
visión. De acuerdo con Burke y Srull (1988), estos efectos se producen porque el primer anuncio del bloque únicamente está afectado por una inhibición retroactiva en el recuerdo; mientras que el último sólo está afectado por una inhibición preactiva. Con este argumento, los spots intermedios ven interferido el recuerdo tanto por la inhibición preactiva como por la retroactiva. Y, por otra parte, la influencia del contexto en el que se inserta el mensaje comercial sobre la memoria, ya que, de acuerdo con la teoría contextual, aquellos elementos que contrastan con su entorno destacarán más y, por lo tanto, tendrán más posibilidades de ser recordados. Así, cuando una persona intenta recordar un determinado elemento (spot o mensaje comercial) el contexto actúa como un facilitador de pistas para dicho recuerdo. Si este elemento está colocado en la primera o última posición del bloque publicitario se incrementa la capacidad de diferenciarse del contexto. Por ello, la posición ocupada por el spot afecta a la capacidad para diferenciar los mensajes del contexto en el cual se insertan (Baddeley, 1990).

Una variable que está muy relacionada con la posición del anuncio en el bloque publicitario es la posición que ocupa el bloque en la parrilla televisiva, es decir, si el bloque publicitario en el que se ha emitido la pieza se sitúa en el corte de un programa o se sitúa en el corte entre programas. A este respecto, varios trabajos han demostrado que los programas en los que se inserta la publicidad pueden afectar a la forma en la que se procesan los anuncios, como es el trabajo de De Pelsmacker, Geuens y Anckaert (2002). Esos trabajos se sustentan en el hecho de que las reacciones de la audiencia hacia el programa no cesan cuando éste se interrumpe por un bloque publicitario; al contrario, éstas se trasladan hacia el mensaje publicitario. La literatura ha estudiado dos posibilidades, la inserción del anuncio en un bloque publicitario durante los intermedios del programa o al final del mismo (bloques publicitarios entre programas). De acuerdo con los trabajos de Chowdhury, Finn y Olsen (2007) y Moorman, Neijens y Smit (2007), el recuerdo que generan los anuncios es mayor cuando se inserta en el intermedio del programa que al final.

Por último, otra de las variables de control consideradas es el efecto repetición. La literatura ha contrastado ampliamente su influencia en la eficacia publicitaria, así como la necesidad de realizar ciertas repeticiones para que produzca sus efectos. Concretamente, los trabajos de Batra y Ray (1986) y Anand y Sterthal (1990), o el más reciente de Yaveroglu y Donthu (2008), relacionan la repetición con un mayor recuerdo.

\section{Metodología de investigación}

\subsection{Datos}

Para alcanzar los objetivos de este estudio se utilizaron fuentes de información primarias y secundarias. Dado que la variable dependiente era el porcentaje de recuerdo espontáneo de marca se realizó un primer estudio de naturaleza primaria para conocer el nivel de recuerdo de una serie de estímulos publicitarios emitidos en televisión a los que los participantes fueron expuestos en un contexto real.

Desde un punto de vista metodológico, uno de los puntos fuertes de este trabajo es que la investigación se ha realizado en un entorno real, tal y como se recomienda en algunos trabajos como los de Moorman et al. (2007) y Pieters y Bijmolt (1997), en los que se señala la necesidad de considerar únicamente válidos los trabajos aplicados en un entorno real, ya que es 
imposible hacer generalizaciones sobre datos obtenidos en entornos forzados. Esta investigación precisó de la colaboración de un soporte televisivo (Telecinco, cadena generalista que emite en abierto y con una cuota media de pantalla situada en torno al 13,9\%) para la difusión en una franja horaria de prime time (con una cuota de pantalla según datos de Kantar Media del 28,6\%) de la parrilla objeto de estudio. El periodo de estudio tuvo una duración de 1 hora y 12 minutos y estaba compuesto por dos programas (teleseries), dos microespacios (contenidos informativos, no planificados en la programación), cinco avances de programación de la cadena, dos espacios de autopromoción de la cadena y 56 piezas publicitarias, de las que 50 se correspondían con spots (publicidad convencional) y seis se correspondían con formatos no convencionales (cinco de ellos patrocinios y una telepromoción). De acuerdo con la clasificación de Infoadex, las categorías de producto anunciadas fueron 27 , referidas a 49 marcas.

La población de este estudio la constituyeron individuos mayores de 10 años, que se corresponde con la audiencia de los programas donde se insertaron las piezas publicitarias objeto de estudio. El proceso metodológico se- guido se caracteriza por (1) la utilización de una encuesta personal no autoadmistrada, aplicada a los individuos que estuvieron expuestos a la cadena durante el tiempo completo de emisión de la parrilla analizada de forma natural, sin tener conocimiento previo de que iban a ser sometidos a la aplicación de un cuestionario posteriormente; (2) la aplicación del cuestionario fue realizada, inmediatamente después del visionado de la parrilla de estudio, por un conjunto de encuestadores instruidos al efecto que debían seleccionar a los encuestados entre su propia red de relaciones; (3) la selección de los individuos se realizó en los hogares de los propios encuestadores, a través del muestreo por relaciones, pero respetando cuotas de género y edad de acuerdo a las cifras de audiencia de televisión en España, y (4) los niveles de recuerdo espontáneo de marca se calcularon a partir de las respuestas dadas por los encuestados a una serie de preguntas en las que se les pedía que indicasen qué marcas recordaban haber visto en el período televisivo de estudio, sin dar pista alguna relativa a los sectores a los que pertenecían las marcas que aparecían en la parrilla analizada. Las Tablas 1 y 2 recogen el proceso metodológico y las características de la muestra, respectivamente.

\section{Tabla 1. Ficha técnica del proceso metodológico del primer estudio sobre recuerdo}

\begin{tabular}{|l|l|}
\hline Procedimiento metodológico & Encuestas personales a través de cuestionario estructurado \\
\hline Universo & Individuos mayores de 10 años. \\
\hline Ámbito & Madrid \\
\hline Muestra real & 816 \\
\hline Error muestral & $+3,4$ \\
\hline Nivel de confianza & $95 \%(\mathrm{p}=\mathrm{q}=50 \%)$ \\
\hline
\end{tabular}


Tabla 2 - Distribución de la muestra del primer estudio sobre recuerdo

\begin{tabular}{|c|c|c|c|c|c|}
\hline Características & $\mathbf{N}$ & $\%$ & Características & $\mathbf{N}$ & $\%$ \\
\hline GÉNERO & & & NIVEL DE ESTUDIOS & & \\
\hline Hombre & 319 & 39,0 & Sin estudios & 33 & 4,0 \\
\hline Mujer & 497 & 60,8 & Primarios & 96 & 11,7 \\
\hline EDAD & & & Secundarios & 151 & 18,5 \\
\hline De 10 a 14 años & 38 & 4,6 & Bachiller & 231 & 28,2 \\
\hline De 15 a 19 años & 69 & 8,4 & Universitarios medios & 151 & 18,5 \\
\hline De 20 a 24 años & 173 & 21,1 & Universitarios superiores & 155 & 18,9 \\
\hline De 25 a 34 años & 149 & 18,2 & CLASE SOCIAL & & \\
\hline De 35 a 44 años & 42 & 5,1 & Alta & 112 & 13,7 \\
\hline De 45 a 54 años & 203 & 24,8 & Media-alta & 180 & 22,0 \\
\hline De 55 a 64 años & 108 & 13.2 & Media-media & 358 & 43,8 \\
\hline \multirow[t]{2}{*}{65 años o más } & 34 & 4.2 & Media-baja y baja & 74 & 9,0 \\
\hline & & & Baja & 2 & 0,2 \\
\hline TOTAL & \multicolumn{5}{|c|}{816} \\
\hline
\end{tabular}

De forma paralela se llevó a cabo otra investigación primaria para conocer la notoriedad previa de cada una de las marcas publicitadas utilizadas en el primer estudio. Se utilizó una encuesta personal autoadministrada siguiendo las recomendaciones de Beerli y Martín (1999), a partir de la cual se calcularon los niveles de notoriedad espontánea (porcentaje total de personas que han nombrado la marca sin sugerencia alguna por parte del entrevistador) y sugerida (porcentaje de personas que reconocen conocer una marca cuando se les menciona) de cada una de las marcas analizadas, en una muestra cuyo perfil, respecto a las variables género y edad, fue similar al de la primera investigación. Concretamente, en el cuestionario diseñado al respecto se pedía al encuestado (1) las marcas que, de forma espontánea, conocía en cada uno de los sectores que aparecían en la parrilla del primer estudio sobre el recuerdo, y (2) que señalase en una lista cerrada en la que figuraban las diferentes marcas que operan en los sectores analizados, aquellas marcas que conocían. Las Tablas 3 y 4 recogen el proceso metodológico y las características de la muestra de este segundo estudio, respectivamente. 


\section{Tabla 3 • Ficha técnica del proceso metodológico del segundo estudio sobre Notoriedad previa}

\begin{tabular}{|l|l|}
\hline Procedimiento metodológico & Encuestas personales a través de cuestionario estructurado \\
\hline Universo & Individuos mayores de 14 años \\
\hline Ámbito & Madrid \\
\hline Muestra real & 192 \\
\hline Error muestral & +7 \\
\hline Nivel de confianza & $95 \%(\mathrm{p}=\mathrm{q}=50 \%)$ \\
\hline
\end{tabular}

\section{Tabla 4 - Distribución de la muestra del segundo estudio sobre Notoriedad previa}

\begin{tabular}{|c|c|c|c|c|c|}
\hline Característica & $\mathbf{N}$ & $\%$ & Característica & $\mathbf{N}$ & $\%$ \\
\hline Género & & & Edad & & \\
\hline Hombre & 89 & 46,4 & De 18 a 24 años & 77 & 40,1 \\
\hline \multirow[t]{5}{*}{ Mujer } & 96 & 50,0 & De 25 a 34 años & 25 & 13,0 \\
\hline & & & De 35 a 44 años & 23 & 12,0 \\
\hline & & & De 45 a 54 años & 35 & 18,2 \\
\hline & & & De 55 a 64 años & 20 & 10,4 \\
\hline & & & 65 años o más & 5 & 2,6 \\
\hline TOTAL & & & 192 & & \\
\hline
\end{tabular}

A partir de la información recabada en los dos estudios anteriores, junto con datos obtenidos de fuentes secundarias, se creó una base de datos que contenía para cada pieza publicitaria la siguiente información: (1) identificación de la pieza publicitaria en términos de categoría de producto, marca y nombre de la campaña; (2) duración de la pieza en segundos; (3) posición de la pieza en el bloque publicitario; (4) número de inserciones de la pieza en la parrilla analizada; (5) posición del bloque en la parrilla, que toma el valor 1 cuando el bloque publicitario en el que se insertó la pieza estaba en el corte de un programa y 0 si estaba en el corte entre programas; (6) notoriedad total de la marca, medida como el porcentaje de personas que conocían de forma espontánea o sugerida la marca anunciada; (7) inversión relativa publicitaria en televisión del 
sector en el que opera la marca anunciada cifrada en millones de euros y creada a partir de los datos de Infoadex (2010). Para ello se procedió a calcular una variable cociente entre la inversión publicitaria en el medio televisión por parte de las marcas que operan en cada uno de los sectores de las marcas anunciadas en las piezas publicitarias objeto de análisis y el número de anunciantes en televisión de cada sector cifrada en millones de euros en el año 2010. Se trata de una variable que puede ser considerada como una proxis de la presión publicitaria que ejerce la marca, ya que no se disponía de la inversión en televisión de todas y cada una de las marcas analizadas, y (8) porcentaje de recuerdo espontáneo de la marca anunciada en la pieza publicitaria.

\subsection{Variables}

Como variable dependiente se utilizó el porcentaje de recuerdo espontáneo de la marca anunciada de las piezas analizadas. Dada su enorme variabilidad, se aplicó la transformación logarítmica para conseguir que la variable se comportara según una distribución normal ( $Z$ de Kolmogorov-Smirnov $=1,369, \mathrm{p}=0,047$ para la variable inicial; $Z$ de Kolmogorov-Smirnov = $0,642, \mathrm{p}=0,804$ para la variable transformada). Como variables independientes se utilizaron las siguientes: (1) la notoriedad total de la marca y (2) la inversión relativa publicitaria en televisión del sector en el que opera la marca. Y, finalmente, como variables de control se consideraron cuatro factores relativos a la planificación publicitaria (1) la duración de la pieza en segundos, (2) la posición de la pieza en el bloque, (3) el número de inserciones de la pieza en la parrilla analizada y (4) la posición del bloque en el programa/parrilla.

\section{Resultados}

Como uno de los problemas presentes en cualquier modelo de regresión es la multicolinealidad se determinó a priori su existencia analizando (1) la correlación entre las variables continuas, (2) el factor de inflación de la varianza (FIV) y (3) la tolerancia. De la Tabla 5, en la que se recogen las correlaciones entre las variables continuas utilizadas en los modelos de regresión, se desprende que existe validación discriminante, ya que ninguno de los coeficientes de correlación es igual a la unidad, asumiéndose, por consiguiente, la no existencia de multicolinealidad. De hecho, la mayoría de los coeficientes de correlación no superan el 0,4. A esta misma conclusión se llega si se observan los valores del FIV y los niveles de tolerancia, ya que los valores FIV no superan el umbral de 10 y los de tolerancia son superiores a 0,10 (véase Tabla 6).

Para lograr el objetivo de esta investigación se utilizó el análisis de regresión jerárquica, en el que se van introduciendo las variables en bloques sucesivos (véase Tabla 6). De esta forma, el modelo I recoge únicamente los efectos de las cuatro variables de control sobre la variable dependiente, es decir, el efecto de la duración, de la posición de la pieza en el bloque, del número de inserciones y de la posición del bloque en el recuerdo publicitario. El modelo II recoge, además de las cuatro variables de control, la variable independiente relativa a la notoriedad de marca, con el objetivo de evaluar su poder explicativo en el recuerdo publicitario de una campaña de forma aislada. Finalmente, el modelo III se diferencia del modelo II únicamente por la inclusión de la variable independiente «Inversión relativa», al objeto de conocer el poder explicativo adicional de la misma en el porcentaje de recuerdo de marca y cuantificar así el esfuerzo diferencial de 


\section{Tabla 5 - Correlaciones entre variables continuas}

\begin{tabular}{|c|c|c|c|c|c|c|c|c|}
\hline Variables & Media & $\begin{array}{l}\text { Desviación } \\
\text { Típica }\end{array}$ & 1 & 2 & 3 & 4 & 5 & 6 \\
\hline 1 Ln Porcentaje Rdo -Marca & 1,35 & 1,34 & - & & & & & \\
\hline 2 Duración & 2,40 & 11,87 & $0,425^{* * *}$ & -- & & & & \\
\hline 3 Posición pieza en bloque & 8,60 & 5,56 & $-0,408^{* * *}$ & $-0,234$ & -- & & & \\
\hline 4 Número inserciones & 1,22 & 0,42 & $0,323^{* *}$ & $-0,228$ & $-0,032$ & --- & & \\
\hline 5 Notoriedad & 86,53 & 19,64 & $0,449^{* * *}$ & $0,310^{* *}$ & $-0,333^{* *}$ & 0,040 & -- & \\
\hline 6 Inversión relativa & 0,36 & 0,12 & $0,649^{* * *}$ & $0,277^{*}$ & $-0,356^{* *}$ & 0,234 & $0,324^{* *}$ & -- \\
\hline
\end{tabular}

NOTA: ${ }^{*} p<0,1,{ }^{* *} p<0,05,{ }^{* * *} p<0,01$

una marca en términos de inversión en la eficacia de sus campañas en términos cognoscitivos.

Los resultados del Modelo I, recogidos en la segunda columna de la Tabla 6, indican, como era de esperar, que el porcentaje de recuerdo de marca de un anuncio es (1) mayor cuanto mayores sean la duración de la pieza publicitaria ( $B=0.429, p=0.001$ ) y la repetición de la misma ( $\beta=0.403, p=0.001$ ) y (2) menor cuanto más avanzada sea la posición de la pieza en el bloque publicitario ( $\beta=-0.332, \mathrm{p}=0.006$ ) y cuando el bloque en el que se ha emitido la pieza sea un bloque emitido entre programas de la cadena ( $ß=-0.217, p=0.052$ ).

De los resultados del modelo II, recogidos en la tercera columna de la Tabla 6, se desprende que, con la incorporación en el modelo I de la notoriedad como variable explicativa del recuerdo, se ha producido un cambio significativo en el coeficiente de determinación y el estadístico $F$ ( $\Delta R 2=9,0 \% ; \Delta F=9,394 ; p=0,004)$, lo que evidencia claramente el efecto positivo y significativo de la notoriedad previa de la marca en el por- centaje de recuerdo de marca $(B=0.336$, $p=0,004)$ y, por consiguiente, la confirmación de la hipótesis $\mathrm{Hl}$.

Para analizar si la inversión relativa publicitaria en televisión en el sector en el que opera la marca presenta un poder explicativo adicional del porcentaje de recuerdo de marca, se estimó el modelo III. Los resultados, recogidos en la cuarta columna de la Tabla 6, muestran que este modelo ha mejorado de forma significativa respecto del modelo II $(\Delta R 2=10,5 \% ; \Delta F=14,474$; $p=0,000) y$, por tanto, que esta nueva variable ejerce una influencia positiva en los porcentajes de recuerdo ( $\beta=0.381, p=0,000$ ). Estos resultados confirman la hipótesis H2 y afirmar que el beneficio de la inversión publicitaria de una marca en los medios por encima de sus competidores tiene una influencia adicional en la eficacia de campañas posteriores.

Finalmente, para comprobar la normalidad de los residuos y garantizar la fiabilidad y validez de los resultados, se aplicó el test de Z de Kolmogorov-Smirnov a los residuos no estandarizados 
Tabla 6. Resultados de los modelos de regresión

\begin{tabular}{|c|c|c|c|c|c|c|c|c|}
\hline \multirow{2}{*}{$\begin{array}{l}\text { Variables } \\
\text { independientes }\end{array}$} & \multicolumn{2}{|c|}{ Modelo I } & \multicolumn{2}{|c|}{ Modelo II } & \multicolumn{2}{|c|}{ Modelo III } & \multicolumn{2}{|c|}{ Colinealidad } \\
\hline & $\begin{array}{l}\text { Beta } \\
\text { estand. }\end{array}$ & $\begin{array}{c}t \\
(p)\end{array}$ & $\begin{array}{l}\text { Beta } \\
\text { estand. }\end{array}$ & $\begin{array}{c}t \\
(p)\end{array}$ & $\begin{array}{l}\text { Beta } \\
\text { estand. }\end{array}$ & $\begin{array}{c}t \\
(p)\end{array}$ & FIV & $\begin{array}{c}\text { Toleran- } \\
\text { cia }\end{array}$ \\
\hline Constante & & $\begin{array}{l}-0,236 \\
(0,814)\end{array}$ & & $\begin{array}{l}-2,311 \\
(0,026)\end{array}$ & & $\begin{array}{l}-3,990 \\
(0,000)\end{array}$ & & \\
\hline Duración & 0,429 & $\begin{array}{r}3,750 \\
(0,001)\end{array}$ & 0,340 & $\begin{array}{r}3,131 \\
(0,003)\end{array}$ & 0,265 & $\begin{array}{r}2,737 \\
(0,009)\end{array}$ & 1,290 & 0,775 \\
\hline $\begin{array}{l}\text { Posición pieza } \\
\text { en bloque }\end{array}$ & $-0,322$ & $\begin{array}{l}-2,906 \\
(0,006)\end{array}$ & $-0,238$ & $\begin{array}{l}-2,257 \\
(0,029)\end{array}$ & $-0,121$ & $\begin{array}{l}-1,248 \\
(0,219)\end{array}$ & 1,288 & 0,777 \\
\hline Número inserciones & 0,403 & $\begin{array}{r}3,637 \\
(0,001)\end{array}$ & 0,378 & $\begin{array}{r}3,708 \\
(0,001)\end{array}$ & 0,284 & $\begin{array}{r}3,075 \\
(0,004)\end{array}$ & 1,172 & 0,853 \\
\hline Posición del bloque & $-0,217$ & $\begin{array}{r}-2,001 \\
(0,052)\end{array}$ & $-0,292$ & $\begin{array}{l}-2,848 \\
(0,007)\end{array}$ & $-0,200$ & $\begin{array}{l}-2,164 \\
(0,036)\end{array}$ & 1,177 & 0,850 \\
\hline Notoriedad & & & 0,336 & $\begin{array}{r}3,065 \\
(0,004)\end{array}$ & 0,316 & $\begin{array}{r}3,296 \\
(0,002)\end{array}$ & 1,263 & 0,792 \\
\hline Inversión relativa & & & & & 0,381 & $\begin{array}{r}3,804 \\
(0,000)\end{array}$ & 1,380 & 0,725 \\
\hline R2 corregido & 0,454 & & 0,541 & & 0,651 & & & \\
\hline $\begin{array}{l}F \\
P\end{array}$ & $\begin{array}{l}10,958 \\
0,000\end{array}$ & & $\begin{array}{l}12,318 \\
0,000\end{array}$ & & $\begin{array}{l}15,893 \\
0,000\end{array}$ & & & \\
\hline$\triangle R 2$ & & & 0,090 & & 0,105 & & & \\
\hline $\begin{array}{l}\Delta F \\
P\end{array}$ & & & $\begin{array}{l}9,394 \\
0,004\end{array}$ & & $\begin{array}{l}14,474 \\
0,000\end{array}$ & & & \\
\hline
\end{tabular}

de los modelos II y III obteniéndose como resultado la total normalidad de las perturbaciones $(Z$ de Kolmogorov-Smirnov=0,823, $p=0,507$ para modelo II y $Z$ de Kolmogorov-Smirnov=0,862, $p=0,448$ para modelo III).

\section{Conclusiones}

Este trabajo, en el que se ha asumido como premisa de partida la consideración del valor de la marca como fuente de ventaja competitiva en el que la notoriedad de marca constituye una de sus dimensiones, tiene como objetivo principal analizar el poder explicativo de la notoriedad previa de una marca en la eficacia de las campañas publicitarias en televisión medida en términos de recuerdo espontáneo de marca. El logro de este objetivo ha proporcionado evidencias empíricas que demuestran que, por un lado, el grado de notoriedad previa de una marca afecta positivamente al recuerdo de sus campañas posteriores, y, por otro, que la presión publicitaria en el medio televisión por parte del sector en el que opera la marca también ejerce una influencia positiva en los porcentajes de recuerdo de la 
marca. La importancia de este estudio es clave en la actual situación del medio televisión que está provocando una pérdida de eficacia general de la publicidad, que ha propiciado la necesidad de plantear el papel que tienen actualmente estas variables sobre el recuerdo, el cual, a su vez, se puede ver afectado por la existencia de otros factores considerados de forma parcelada en la literatura, y que en nuestro estudio se han considerado conjuntamente como variables de control.

En cuanto a los efectos de las variables de control sobre el recuerdo, los resultados son congruentes con los recogidos en la literatura y con la praxis empresarial. Así, en primer lugar, se contrastó que el porcentaje de recuerdo de marca de un anuncio es mayor cuanto mayor es su duración y su nivel repetición. El efecto de la duración sobe el recuerdo fue también contrastado por Bigné y Miquel (1996), entre otros, y se relaciona con el mayor nivel de aprendizaje conseguido de dichos contenidos. De ahí que este resultado se pueda justificar desde la teoría del aprendizaje, la cual afirma que la cantidad de información recordada es una función directa del tiempo total dedicado al aprendizaje (Singh y Cole, 1993). En cuanto a la repetición, nuestro resultado coincide con el obtenido en los trabajos de Batra y Ray (1986), Anand y Sterthal (1990) o Yaveroglu y Donthu (2008), en los que la repetición de un spot genera un mayor recuerdo. Frente a los problemas de eficacia que presenta actualmente la saturación publicitaria y los resultados negativos sobre las actitudes que puede producir la excesiva repetición del anuncio, recogidos en el trabajo de Campbell y Keller (1993), en el medio televisión los resultados obtenidos permiten confirmar al menos la buena relación frecuencia de contacto/recuerdo que permite el medio, y cómo es preciso realizar cier- tas repeticiones de un estímulo publicitario para que éste sea recordado. Y, en segundo lugar, los resultados han arrojado que el recuerdo es menor cuanto más avanzada sea la posición de la pieza en el bloque publicitario y cuando el bloque en el que se ha emitido la pieza es un bloque emitido entre programas. Este resultado tiene su explicación, por una parte, en la existencia de un proceso de interferencia entre los diferentes mensajes publicitarios, que explicaría el efecto primacía obtenido en nuestro estudio, que se produce, de acuerdo con Burke y Srull (1988), porque el primer anuncio del bloque únicamente está afectado por una inhibición en el recuerdo retroactiva y el último anuncio del bloque sólo por una inhibición en el recuerdo preactiva. Y, por otra parte, y en línea con el trabajo de Baddeley (1990), en que la posición ocupada por el spot afecta a la capacidad para diferenciar los mensajes del contexto en el cual se insertan. En este contexto, los planificadores de medios asumen que la selección de los programas en los que se inserta la publicidad no es sólo una decisión relacionada con el volumen de audiencia y con la estructura sociodemográfica de la misma (Kimms y Muller-Bungart, 2007), ya que el programa, en sí mismo, es un contexto que afecta al impacto del mensaje. Es por ello que la influencia del contexto en el que se inserta la publicidad sea una cuestión de creciente interés en un entorno de pérdida de eficacia como el que presenta actualmente el medio televisivo. A este respecto, nuestros resultados, de acuerdo con Chowdhury et al. (2007), indican que los efectos del programa sobre la publicidad son más fuertes en un anuncio situado en el intermedio del programa.

En cuanto a la notoriedad, los resultados confirman que se trata de una fuente de ventaja competitiva a la que se añade una nueva exten- 
sión a las proporcionadas por la literatura, dado los beneficios que aporta a una empresa en términos de eficacia publicitaria.

Como implicación práctica de este trabajo, y a pesar de los condicionantes negativos relacionados con el actual entorno competitivo del medio televisivo, se observa como la televisión es un medio efectivo a la hora de lograr objetivos comunicacionales de los anunciantes en términos de recuerdo. De hecho, las marcas que la utilizan en su plan de medios persiguen, entre sus objetivos, generar altos niveles de notoriedad y que esa notoriedad sea constante en el tiempo. Estos resultados ponen en evidencia investigaciones promovidas por la industria publicitaria (Media Planning Group, 2007) que cuestionan, ante los condicionantes asociados al medio televisión, que se mantenga la relación positiva entre notoriedad previa de una marca y eficacia publicitaria; ya que, como se ha demostrado, las empresas deben velar por sus tasas de notoriedad en la medida en que ayudan a conseguir y reforzar una mayor eficacia de sus campañas posteriores.

Desde un punto de vista metodológico, el valor de este trabajo para la gestión se deriva del hecho de evaluar la componente cognitiva de la eficacia publicitaria utilizando el recuerdo espontáneo de marca, ya que tal y como señalan De Pelsmacker, Geuens y Van Den Bergh (2005) es una medida nemotécnica más fiable y por ello más difícil de obtener frente al recuerdo sugerido o al reconocimiento más habitualmente utilizados. En este sentido, es frecuente encontrar, especialmente en la praxis de los institutos de investigación, trabajos que sólo presentan cifras de recuerdo asistido y/o reconocimiento, dado los bajísimos niveles que genera el recuerdo publicitario espontáneo en trabajos desarrollados en un entorno real.

Las principales limitaciones de este trabajo se circunscriben, por una parte, y a pesar de que la muestra utilizada es representativa del perfil de la audiencia de televisión, a la realización del estudio en Madrid. En futuros trabajos sería conveniente replicar esta investigación a nivel nacional. Y, por otra parte, en el presente trabajo sólo se ha analizado el efecto de la notoriedad sobre el recuerdo publicitario, cuando, los diferentes modelos de respuesta publicitaria clasifican los efectos publicitarios en cognitivos, afectivos y conativos como base para medir la eficacia de la publicidad. Por ello, en futuros trabajos resultaría interesante continuar la investigación con el análisis de los efectos de la notoriedad a niveles afectivos y conativos. Finalmente, sería de interés para el avance en la investigación sobre el recuerdo publicitario, analizar los efectos interactivos de las variables de planificación de medios, abordadas en este estudio como variables de control, de cara a la optimización de las decisiones de planificación. 


\section{Bibliografía}

Aaker, D.A. (1996). Managing Brand Equity Capitalizing on the Value of Brand Name. New York: The Free Press.

Aaker, D.A., Batra, R. y Myers, J.G. (1992). Advertising management. New Jersey: Prentice Hall.

Anand, P. y Sterthal, B. (1990). Ease of message processing as a moderator of repetition effects in advertising. Journal of Marketing Research, 27(3), 345-353.

Baddeley, A.D. (1990). Human Memory: Theory and Practice. Boston: Allyn \& Bacon.

Balasubramanian, S.K., Karrh, J.A. y Patwardhan, H. (2006). Audience Response to Product Placements: An Integrative Framework and Future Research Agenda. Journal of Advertising, 35(3), 115-141.

Batra, R. y Ray, M.L. (1986). Situational Effects of Advertising Repetition: The Moderating Influences of Motivation, Ability, and Opportunity to Respond. Journal of Consumer Research, 12(4), 432-445.

Beerli, A. y Martín, J.D. (1999). Técnicas de medición de la eficacia publicitaria. Barcelona: Ariel.

Bendixen, M.T. (1993). Advertising effects and effectiveness. European Journal of Marketing, 27(10), 19-32.

Bigné, E. y Miquel, M.J. (1996). La duración del anuncio: efectos cognitivos y afectivos. En Luque, T. (Ed.). La empresa en una economía globalizada: retos y cambios, Vol. IB. (pp.767-778). Granada: Asociación Europea de Dirección y Economía de la Empresa.

Burke, R. y Srull, T.K. (1988). Competitive Interference and Consumer Memory for Advertising. Journal of Consumer Research, 15(1), 55-68.

Campbell, M.C. y Keller, K.L. (2003). Brand Familiarity and Advertising Repetition Effects. Journal of Consumer Research, 30 (septiembre), 292-304.

Chowdhury, R., Finn, A. y Olsen, G.D. (2007). Investigating the Simultaneous Presentation of Advertising and Television Programming. Journal of Advertising, 36(3), 85-96.

Davies, G. y Rojas-Mendez, J.I. (2005). Avoiding Television Advertising: Some Explantions from Time Allocation Theory. Journal of Advertising Research, 45(1), 34-48.

De Pelsmacker P., Geuens, M. y Anckaert P. (2002). Media context and advertising effectiveness: the role of context style, context quality and context-ad similarity. Journal of Advertising, 31(2), 49 -61.

De Pelsmacker, P., Geuens, M. y Van Den Bergh, J. (2005). Marketing Communicatie. Amsterdam: Pearson Education.

Del Río Lanza, A.B., Iglesias Argüelles, V. y Vázquez Casielles, R. (2000). La influencia de la marca sobre el comportamiento del consumidor y el efecto moderador de su orientación hacia las marcas comerciales. Revista Española de Investigación y Marketing, 4(2), 51-79.

Del Río Lanza, A.B., Iglesias Argüelles, V. y Vázquez Casielles, R. (2002). El valor de la marca: Perspectivas de análisis y criterios de estimación. Cuadernos de Gestión, 1(2), 87102.

Dubow, J.S. (1994). Point of view: Recall revisited: Recall redux. Journal of Advertising Research, 34(3), 92-99.

Fabian, G.S. (1986). 15-second commercials: The inevitable evolution. Journal of Advertising Research, 26(4), R C3-5. Feldwick, P. (1996). What is Brand Equity anyway, and how do you measure it? Journal of Market Research Society, 38(2), 85-104.

Gázquez, J.C., Jiménez, D., Sáez, E. y Sánchez, M. (2010). El comportamiento de cambio de canal del telespectador en prime time. Análisis a través de un panel de audiometría. Revista Española de Investigación de Marketing, 14(2), 31-66.

Carrero, E. y González, M. J. (2008). Manual de Planificación de Medios. Madrid: Esic.

Higie, R.A. y Sewall, M.A. (1991). Using recall and brand preference to evaluate advertising effectiveness. Journal of Advertising Research, 31(2), 56-63.

Hoyer, W.D. y Brown, S.P. (1990). Effects of brand awareness on choice for a common, repeat purchase product. Journal of Consumer Research, 17(2), 141-148.

Infoadex (2010). Estudio Infoadex de la inversión publicitaria en España 2010. Madrid: Infoadex.

Jamhouri, O. y Winiarz, M. (2009). The enduring influence of TV advertising and communications clout patterns in the global marketplace. Journal of Advertising Research, 49(2), 227-235.

Kantar Media (2011). Anuario de audiencias de televisión 2011. España: Kantar Media Audiences. 
Keller, K.L. (1993). Conceptualizing, Measuring, and Managing Customer-Based Brand Equity. Journal of Marketing, 57(1), 1-22.

Keller, K.L. y Aaker, D.A. (1997). Managing the Corporate Brand: The Effect of Corporate Marketing Activity on Consumer Evaluations of Brand Extensions. Working paper 97-106. Marketing Science Institute.

Kent, R.J. y Allen, C.T. (1994). Competitive Interference Effects in Consumer Memory for Advertising: The Role of Brand Familiarity. Journal of Marketing, 58(3), 97-105.

Kimms, A. y Muller-Bungart, M. (2007). Revenue management for broadcasting commercials: the channel's problem of selecting and scheduling the advertisements to be aired. International Journal of Revenue Management, 1(1), 28-44.

Kotler, P. y Keller, K.L. (2007). Marketing management. New Jersey: Prentice Hall

Lamas, C. (2004, febrero). Saturación, concentración, multimedia: algunos condicionantes de la eficacia de las campañas. Ponencia presentada al $20^{\circ}$ AEDEMO de Televisión, Santiago de Compostela, España.

Lambin, J.J. (1995). Marketing estratégico. Madrid: McGrawHill.

Li, C. (2010). Primacy effect or recency effect? A long-term memory test of Super Bowl commercials. Journal of Consumer Behaviour, 9(1), 32-44.

Lowrey, T.M., Shrum, L. J. y Mccarty, J.A. (2005). The Future of Television Advertising. En Kimmel, A.J. (Ed.). Marketing Communication: Emerging Trends and Developments (pp. 113-132). New York: Oxford University Press.

Macdonald, E. y Sharp, B. (2003). Management Perceptions of the Importance of Brand Awareness as an Indication of Advertising Effectiveness. Marketing Bulletin, 14 (mayo), 1-11

Mediaedge (2008). Panorama de la Televisión en España 2007. Madrid: Mediaedge Cía.

Media Planning Group (2007). Eficacia publicitaria de las acciones publicitarias especiales en televisión. España: Media Planning Group.

Mela, C.F., Gupta, S. y Lehmann, D.R. (1997). The LongTerm Impact of Promotion and Advertising on Consumer Brand Choice. Journal of Marketing Research, 34(2), 248-261.

Moorman, M., Neijens, P.C. y Smit, E. (2005). The Effects of Program Responses on the Processing of Commercials
Placed at Various Positions in the Program and the Block. Journal of Advertising Research, 45(1), 49-59.

Moorman, M., Neijens, P.C. y Smit, E. (2007). The Effects of Program Involvement on Commercial Exposure and Recall in a Naturalistic Setting. Journal of Advertising, 36(1), 121-137.

Newell, S.J., Henderson, K.V. y Wu, B.T. (2001). The Effects of Pleasure and Arousal on Recall of Advertisements during the Super Bowl. Psychology and Marketing, 18(11), 35-53.

Perreault, J.D. y Pettigrew, D. (1998). Bilan de mesures d`efficacité publicitaire utilisées dans les agences de publicité québécoises. Revue Français du Marketing, 166, 69-75.

Pieters, R. y Bijmolt, T. (1997). Consumer Memory for Television Advertising: A Field Study of Duration, Serial Position, and Competition Effects. Journal of Consumer Research: An Interdisciplinary Quarterly, 23(4), 362-372.

Pieters, R., Warlop, L. y Wedel, M. (2002). Breaking Through the Clutter: Benefits of Advertisement Originality and Familiarity for Brand Attention and Memory. Management Science, 48(6), 765-781.

Reinares, P. (2010). Jóvenes y Televisión generalista en España: ¿es Internet responsable de una audiencia perdida?. Revista de Estudios de Juventud, 88, 63-75.

Rossiter, J.R. y Percy, L. (1997). Advertising communications and promotion management. New York: McGraw-Hill.

Rubinson, J. (2009). Empirical evidence of TV advertising effectiveness. Journal of Advertising Research, 49(2), 220-226. Ruževičiūté, R. y Ruževičius, J. (2010). Brand equity integrated evaluation model: consumer-based approach. Economics and Management, 15, 719-725.

Shapiro, S. y Krishnan, S. (2001). Memory-based Measures for Assessing Advertising Effects: A Comparison of Explicit and Implicit Memory Effects. Journal of Advertising, 30(3), $1-13$.

Sharp, B., Beal, V. y Collins, M. (2009). Television: back to the future. Journal of Advertising Research, 49(2), 211-219.

Singh, S.N. y Cole, C.A. (1993). The Effects of Length, Content, and Repetition on Television Commercial Effectiveness. Journal of Marketing Research, 30(1), 91-104.

Villarejo Ramos, A.F. (2002). La medición del valor de marca en el ámbito de la gestión de Marketing. Sevilla: Ceade. 
Wells, W.D., Burnett, J. y Moriarty, S. (2005). Advertising. Principles and practice. New Jersey: Prentice Hall.

Woltman Elpers, J.L.C.M., Wedel, M. y Pieters, R.G.M. (2003). Why do consumers stop viewing television commercials? Two experiments on the influence of moment-tomoment entertainment and information value. Journal of Marketing Research, 40(4), 437-453.

Yaveroglu, I. Y Donthu, N. (2008). Advertising repetition and placement issues in on line environments. Journal of Advertising, 37(2), 31-43.
Zhao, X. (1997). Clutter and serial order redefined and retested. Journal of Advertising Research, 37(5), 57-73.

Zenith (2013). Los Medios en España y Portugal 2012. España: Zenith.

Zhielske, H.A. (1982). Does day-after recall penalize «Feeling ads». Journal of Advertising, 22(1), 11-22. 